This article shows how Cox models can be used to study multiepisode-multistate processes as well as which computer programs can be used to estimate semiparametric models with multispell data. The application of a multiepisode Cox model is demonstrated using data on career trajectories of German males from the German Life History study.

\title{
Using Cox Models to Study \\ Multiepisode Processes
}

\author{
HANS-PETER BLOSSFELD \\ Max Planck Institute for \\ Human Development and Education \\ ALFRED HAMERLE \\ University of Konstanz
}

\begin{abstract}
INTRODUCTION
In recent years, more and more social scientists have used continuous-time models and event history data to study economic and social processes. This approach has led to the use of general multistate-multiepisode models. Most of the available software (e.g., BMDP, SAS, GLIM), however, is designed to deal with single-spell models. When reducing the analysis of multiple-spell data to the analysis of single-spell models, some restrictive assumptions are usually made. For example, it is assumed that (1) duration distributions only depend on the time from entry to the present state, (2) successive episodes of an individual are independent, and (3) the effects of the

AUTHORS' NOTE: This paper results from work undertaken as part of the West German life history project, which is based in the Max Planck Institute for Human Development and Education and supported by grants of the Deutsche Forschungsgemeinschaft. For helpful comments on an earlier draft, we wish to thank Michael T. Hannan, Karl Ulrich Mayer, Kevin Selbee, Jaqui Smith, and the anonymous reviewers.
\end{abstract}


explanatory variables are the same for all episodes. In most cases, these assumptions are not stated explicitly. If they are valid, then consecutive spells of an individual may be treated as if they stemmed from different individuals with the corresponding covariate values, and all spells can be analyzed together within the framework of single-spell models and with single-spell programs. Nevertheless, one should keep in mind that the assumptions that allow such a procedure are restrictive and must be examined empirically.

Several authors, including Kalbfleisch and Prentice (1980:chp. 7), Tuma and Hannan (1984), and Heckman and Singer (1984), have studied models for multiepisode duration data without the restrictive assumptions mentioned above. Most of the articles consider special cases, Semi-Markov models in particular. In light of this burgeoning literature, the purpose of this article is fourfold: First, to present a partially parametric model (Cox 1972, 1975) that leaves the form of time variation in multiepisode models unspecified but makes the "baseline" hazard rate dependent on the number of the episode; second, to investigate the implications of different time scales that lead to different underlying stochastic processes; third, to discuss to what extent available computer programs can process multispell data; and fourth, to illustrate empirically the specification, estimation, and testing of a multiepisode proportional hazards model in order to study career mobility.

\section{COX MODELS FOR MULTIEPISODE PROCESSES}

Let us suppose that for every individual and episode $k$, a vector $x_{k}$ of relevant covariates is measured. The vector of covariates may contain metric or dummy variables or both. For the moment, assume that the covariates are time invariant, although most of the methods can be generalized to allow for time-dependent covariates. If an individual is in the kth episode, his previous history of the process until $t_{k-1}$ is collected in $\mathrm{H}_{k-1}$ (dropping the subscript $i$ to simplify notation); that is, 


$$
\mathrm{H}_{\mathrm{k}-1}=\left\{\mathrm{y}_{0}, \mathrm{t}_{1}, \mathrm{y}_{1}, \mathrm{x}_{1}, \ldots, \mathrm{t}_{\mathrm{k}-1}, \mathrm{y}_{\mathrm{k}-1}, \mathrm{x}_{\mathrm{k}-1}\right\}
$$

Now we define a new regression vector $z_{k}, k=1,2, \ldots$, which includes $x_{k}$, the covariates measured at the beginning of the kth episode, and all relevant elements of $\mathrm{H}_{\mathrm{k}-1}$. The dimension of $\mathrm{z}_{\mathrm{k}}$ is $\mathrm{p}_{\mathrm{k}}$. The duration process is characterized by a hazard rate (exit rate), and is defined by

$$
\lambda^{k}\left(t \mid z_{k}\right)=\lim _{\Delta t \rightarrow 0} P\left(t \leq T_{k}<t+\Delta t \mid T_{k} \geq t, z_{k}\right) / \Delta t, \quad t \geq t_{k-1}
$$

Note that $\lambda^{k}\left(t \mid z_{k}\right)$ will be identically zero for $t<t_{k-1}$. Equation (1) is the overall exit rate for the kth episode. In addition, "transition specific" exit rates (hazard rates) are defined:

$$
\lambda_{j}^{k}\left(t \mid z_{k}\right)=\lim _{\Delta t \rightarrow 0} P\left(t \leq T_{k}<t+\Delta t, Y_{k}=j \mid T_{k} \geq t, z_{k}\right) / \Delta t
$$

The possibility that some states are not attainable from certain states or that the set of attainable states varies with $\mathrm{k}$ in a more general manner can be accommodated in (2) by restricting it to be identically zero for the appropriate $(k, j)$ values.

The relationship between (1) and (2) is

$$
\lambda^{k}\left(t \mid z_{k}\right)=\sum_{j} \lambda_{j}^{k}\left(t \mid z_{k}\right)
$$

Survivor function and density function of the kth episode can be defined analogously to the single-spell case. For the exact definitions and for the relationship between survivor function, density function, and exit rate, see Blossfeld et al. (1988) or Tuma and Hannan (1984).

For a complete specification of the exit rate, the following must be fixed: (1) the time dependence of the exit or hazard rate, (2) how much of the previous history is included, and (3) the dependence on the incorporated covariates.

In this article, a general proportional hazards model is considered. The proportional hazards model was proposed by Cox (1972), and its hazard or exit rate is 


$$
\lambda(t \mid z)=\lambda_{0}(t) \exp \left(z^{\prime} \beta\right)
$$

In (4), $\lambda_{0}(t)$ is an unspecified baseline hazard rate. In the approach here, $\lambda_{0}(t)$ as well as $\beta$ may depend on the origin state $y_{k-1}$, the destination state $y_{k}$, and the number $k$ of the current episode; that is, $\lambda_{\text {olj }}^{k}(t)$ and $\beta_{1 j}^{k}$ (given that $y_{k-1}=1$ and $y_{k}=j$ ), although in (4) the subscripts have been dropped to simplify notation.

A variety of restrictions could be placed on the baseline exit rates or on the parameters $\beta$. The focus here is on two classes of restrictions, namely, restrictions on time dependence and on the covariate effects. For the estimation of the regression coefficients, a partial likelihood procedure is used. The partial likelihood for the general model factors into a separate component for each 1 and $j$ that can be maximized regarding a specific transition from state 1 to state $j$. Therefore, consider a specific $(1, j)$ transition, dropping the subscripts 1 and $j$.

With regard to time dependence of the hazard rate, only two alternatives are distinguished. In the first approach, the time parameter $t$ means time since the beginning of the process, although only rarely will the time parameter correspond to calendar time. In this article, the time parameter of the process corresponds to time since entry into the labor market, and all subsequent starting and ending times of a person's episodes are calculated as the time since this event. In the second approach, it is assumed that the exit rate only depends on the time since the occurrence of the last transition, that is, on $t-t_{k-1}$. These models are suitable when the exit rates for the duration process can be more parsimoniously expressed in terms of gaps between consecutive spells rather than in terms of total observation time, or the time since entry into the labor market. Semi-Markov processes and renewal processes are special cases. Models of this type are studied by Heckman and Singer (1984) in a statistical context. In the following, only the formulas for the models with time parameter $t$ are given. The models that depend on $t-t_{k-1}$ can be derived analogously.

First, consider the multiepisode model with episode-changing regression coefficients and episode-changing baseline hazards:

$$
\lambda^{k}\left(t \mid z_{k}\right)=\lambda_{o k}(t) \exp \left(z_{k}^{\prime} \beta_{k}\right)
$$


It can be shown (Hamerle, forthcoming) that an appropriate partial likelihood for model (5) is

$$
P L_{1}=\prod_{k} \prod_{i=1}^{d_{k}} \frac{\exp \left(z_{i k}^{\prime} \beta_{k}\right)}{\sum_{r \in R_{k}\left(t_{i k}\right)} \exp \left(z_{r k}^{\prime} \beta_{k}\right)}
$$

where $t_{1 k}, t_{2 k}, \ldots$ are the uncensored durations of the kth episode and $z_{i k}$ is the covariate vector of the individual with duration $t_{i k}$. The risk set $R_{k}(t)$ is the set of individuals at risk of their kth event just before time $t(k-1$ but not $k$ transitions have occurred before $t)$.

If there are no equality restrictions among the $\beta_{\mathrm{k}}$, equation (6) can be maximized separately for each $\mathrm{k}$. For that purpose common computer programs for single-spell models (e.g., BMDP, RATE, GLIM) can be used without difficulties if it is assumed that the baseline hazard rates only depend on the time since entry into the current job, that is, on $t-t_{k-1}$. In single-spell models, the starting point of the spell usually is set to 0 . The risk set then decreases as time elapses. This also holds in multiepisode models provided that time is measured as waiting time in the current state-the clock is reset to 0 after each transition.

If the time scale represents observation time, or the time since the occurrence of an individual specific event such as birth or entry into the labor market, the situation is more complicated. In this case, all subsequent starting and ending times of a person's episodes are calculated as the time since the occurrence of this event. Then, for the $k$ th episode, $k \geq 2$, an individual only belongs to the risk set $R_{k}(t)$ if he is at risk of his kth event at time $t$; that is, $k-1$ but not k events have occurred before time t. Here, the risk set may also increase as time goes on, because in a certain time interval some persons can have their $(\mathrm{k}-1)$ th event and are then at risk of their kth event, although no individual has his kth event in this time interval. The risk set is increasing in this time interval. The situation is illustrated in Figure 1.

The second model investigated here assumes that episode $\mathrm{k}, \mathrm{k}=1$, $2, \ldots$ has a common shape function $\lambda_{\mathrm{o} 1}(\mathrm{t})=\lambda_{\mathrm{o} 2}(\mathrm{t})=\ldots \lambda_{\mathrm{o}}(\mathrm{t})$. The exit rate can then be written as

$$
\lambda^{\mathrm{k}}\left(\mathrm{t} \mid \mathrm{z}_{\mathrm{k}}\right)=\lambda_{\mathrm{o}}(\mathrm{t}) \exp \left(\mathrm{z}_{\mathrm{k}}^{\prime} \beta_{\mathrm{k}}\right)
$$




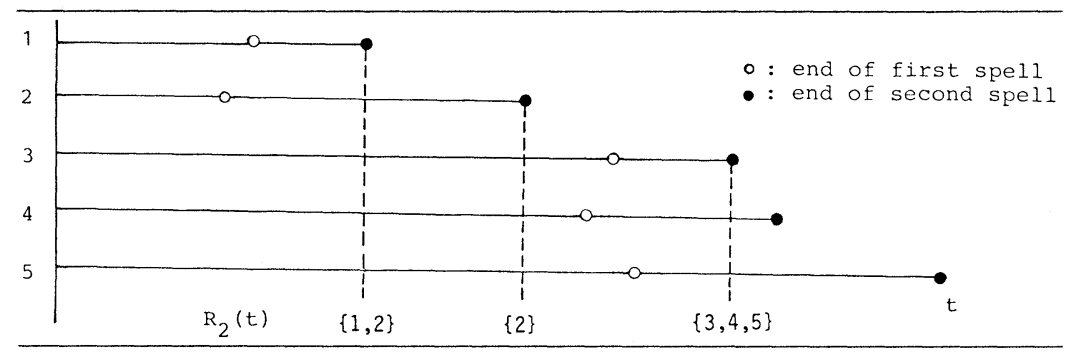

Figure 1: Length of Two Subsequent Episodes, Measured for Five Persons, and Construction of Risk Set $R_{2}(t)$

An appropriate partial likelihood for model (7) is Kalbfleisch and Prentice (1980:184)

$$
P L_{2}=\prod_{k} \prod_{i=1}^{d_{k}} \frac{\exp \left(z_{i k}^{\prime} \beta_{k}\right)}{\sum_{m} \sum_{r \in R_{m}\left(t_{i k}\right)} \exp \left(z_{r m}^{\prime} \beta_{k}\right)}
$$

Because the denominator of (8) contains all $\beta_{\mathrm{k}}, \mathrm{k}=1,2, \ldots$, this partial likelihood does not factor into a separate component for each $\mathrm{k}$, and the coefficients $\beta_{\mathrm{k}}$ will need to be estimated simultaneously.

For estimation of models of this type with programs for single-spell models, a rearrangement of regression vectors and parameter vectors is necessary. Let $\mathrm{K}$ be the maximum number of episodes, and let $\tilde{\mathrm{x}}_{\mathrm{i}}$ denote the covariates that are the same across episodes-for example, sex, race, and demographic variables that remain fixed during the observation period. The covariates of individual $i$ that vary over episodes are denoted by $x_{i 1}, \ldots x_{i n_{i}}, i \leq n_{i} \leq K$. The number of episodes of individual $\mathrm{i}$ in the observation period is $\mathrm{n}_{\mathrm{i}}$. The dimension of $\tilde{x}_{\mathrm{i}}$ is $\mathrm{q}$, the dimension of $\mathrm{x}_{\mathrm{ik}}$ is $\mathrm{q}_{\mathrm{k}}$. Now, each individual new design vector is defined, denoted by $z_{\mathrm{ik}}$ : 


$$
\mathrm{z}_{\mathrm{i} 1}=\left(\begin{array}{l}
\tilde{\mathrm{x}}_{\mathrm{i}} \\
\mathrm{x}_{\mathrm{i} 1} \\
0 \\
\cdot \\
\cdot \\
\cdot \\
0
\end{array}\right), \ldots, \mathrm{z}_{\mathrm{in}_{1}}=\left(\begin{array}{l}
\tilde{\mathrm{x}}_{\mathrm{i}} \\
0 \\
\cdot \\
\cdot \\
\mathrm{x}_{\mathrm{in}} \\
0 \\
\cdot \\
\cdot \\
0
\end{array}\right), \quad 1 \leq \mathrm{n}_{\mathrm{i}} \leq \mathrm{K}
$$

The dimension of the new design vectors $z_{i k}$ is $q+\sum_{k=1}^{K} q_{k}$. Corresponding to the design vectors, a suitably dimensioned parameter vector is defined:

$$
\beta=\left(\begin{array}{l}
\tilde{\beta} \\
\beta_{1} \\
\cdot \\
\cdot \\
\beta_{\mathrm{k}}
\end{array}\right)
$$

Then, with the above definitions, we have

$$
z_{\mathrm{ik}}^{\prime} \beta=\tilde{x}_{\mathrm{i}}^{\prime} \tilde{\beta}+\mathrm{x}_{\mathrm{ik}}^{\prime} \beta_{\mathrm{k}}
$$

With this rearrangement, one can use the data of all episodes, and maximum partial likelihood estimation based on the likelihood expression (8) can be done with a program for single-spell models. Assuming that the time scale is time since the last transition-e.g., entry into the current job-for a given $t_{i k}$, the denominator of (8) contains all spells that last at least $t_{i k}$. There may be several spells for an individual. According to the serial order of the spell, the corresponding $\exp (.$.$) term is chosen; in this case only one risk set is$ needed. Through the special construction of the design vectors $z_{i k}$, the correct $\exp (. .$.$) term is chosen. For the time scale representing the$ time since the occurrence of a specific event, the procedure is similar. 
In this restricted model, tests for equality of some elements of $\beta_{k}$ across episodes can easily be performed using the likelihood ratio statistic, the Wald statistic, or the score statistic. In the third proportional hazards model that is investigated, it is assumed that both the shape functions and the regression coefficients are the same over episodes. The exit rate is then

$$
\lambda^{k}\left(t \mid z_{k}\right)=\lambda_{o}(t) \exp \left(z_{k}^{\prime} \beta\right)
$$

From (8), we have the partial likelihood

$$
\mathrm{PL}_{3}=\prod_{\mathrm{k}} \prod_{\mathrm{i}=1}^{\mathrm{d}_{\mathrm{k}}} \frac{\exp \left(\mathrm{z}_{\mathrm{ik}}^{\prime} \beta\right)}{\sum_{\mathrm{m}} \sum_{\mathrm{r} \in \mathrm{R}_{\mathrm{m}}\left(\mathrm{t}_{\mathrm{i} k}\right)} \exp \left(\mathrm{z}_{\mathrm{rm}}^{\prime} \beta\right)}
$$

But $\mathrm{PL}_{3}$ is equivalent to the partial likelihood that arises if a person's episodes are treated as if they stem from different persons and all episodes are analyzed together with a single-spell model. Here, construction of design vectors $z_{i k}$ of the form (9) is not needed.

Here, only the application of Cox models to the multiepisode case is considered. For the extension of fully parameterized hazard rate models to the multiepisode case and for a further discussion of multiple-spell duration models, see Hamerle (forthcoming).

\section{STUDYING CAREER MOBILITY AS A MULTIEPISODE PROCESS}

As an example of the specification, estimation, and testing of a multiepisode proportional hazards model, the career trajectories of German men from three birth cohorts will be analzyed. In most studies in social mobility research, transitions to a destination job are modeled as a function of the time since entry into an origin job (see Sorensen, 1984; Sorensen and Tuma, 1981; Carroll and Mayer, 1986; Tuma, 1985). The career trajectory of an individual is therefore divided into a series of job spells, each starting with time equal to 0 . In this case, time-dependence means that the event of a job shift primarily depends 
on the time spent in each of these jobs, regardless of their specific locations over a person's life course. Moreover, the effects of causal variables are mostly regarded as independent of the different job episodes, and therefore are estimated as constant across episodes. One can call this type of job-shift analysis "uniepisode models with episode-constant coefficients."

Another way to model job mobility is to regard job transitions as dependent on the general labor-force experience of a person. The time scale in this case is the amount of general labor-force experience. Starting and ending times of the job spells in a person's career are given as the time since entry into the labor market. In this case, time-dependence means that the event of a job shift depends on the specific location of the spell within a person's life course. Consequently, the job spells of a person's career are not treated as autonomous entities, but depend on their specific location on the general laborforce experience scale. The coefficients of causal variables may be estimated as constant or as changing across episodes. One can call this type of job-shift analysis "multiepisode models with episode-constant or episode-changing coefficients."

The empirical analysis of career mobility here is based on life history data collected between October 1981 and May 1983 by Mayer. The life-history data include information from 2,171 German respondents from the cohorts of 1929-1931,1939-1941, and 1949-1951. This sample is representative of the native-born German population of the Federal Republic of Germany (Brückner et al., 1984; Blossfeld, $1987 \mathrm{c}$ ), and constitutes a unique source of information about the occupational histories of men and women from selected birth cohorts. Women are excluded from the present analysis because they show very different occupational structures and mobility patterns.

The empirical example begins with a description of how job spells are distributed across episodes. Table 1 shows that for German men born in 1929-1931, 1939-1941, and 1949-1951, the first two job episodes make up more than $50 \%$ of all spells, and the first five episodes some $90 \%$ of all spells. Compared to American workers, German workers have considerably fewer jobs and change jobs less frequently. The average time in a given job for a man in Germany is 


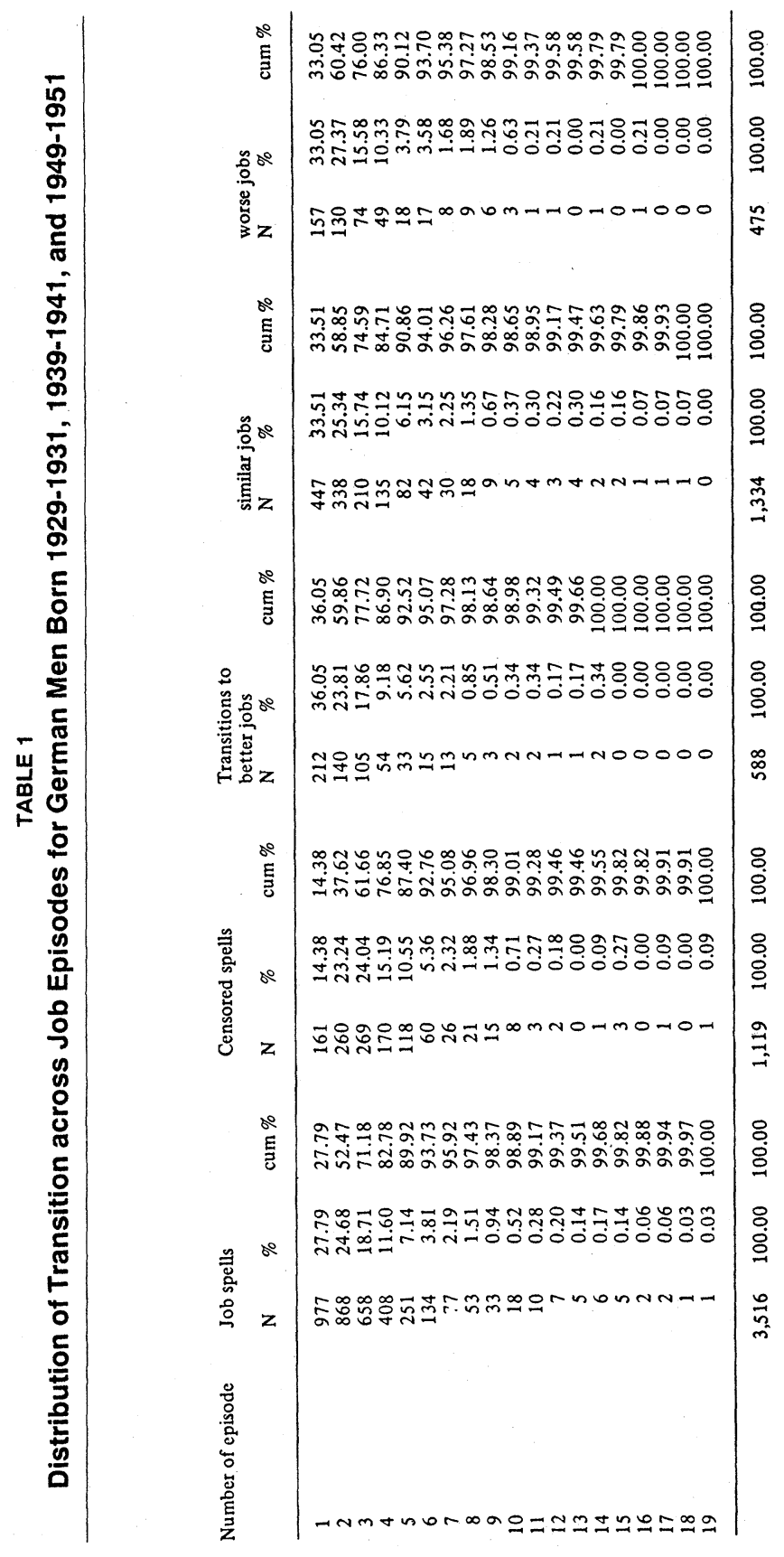


about 5.98 years (Carroll and Mayer, 1986), while in the United States it is only 2.17 years (Tuma, 1985).

The stable nature of job trajectories in Germany implies that job changes are more likely to be substantively meaningful than is job mobility in the United States. But the stable nature of job transitions also means that the distribution of job spells across episodes is very highly skewed toward the early episodes (see Table 1). This has consequences for the analysis of career mobility as a multiepisode process: (1) The smaller the number of job spells and job transitions, the more likely it is to produce statistically insignificant results; hence, a comparison across job episodes based on significant coefficients may be misleading. (2) The number of job episodes used to study career mobility as a multiepisode process is limited. In such a model, it is not sensible to include episodes with very small numbers of job spells and job transitions. Therefore, based on the distribution of job spells and job transitions across episodes, as displayed in Table 1, only the first five job episodes are included in this multiepisode model. This ensures that the number of job spells and transitions in each episode is great enough to compare the estimates across episodes in a meaningful way. Additionally, it guarantees that few job spells are lost in the analysis. On the basis of this decision, included in this multiepisode model are $89.92 \%$ of all job spells, $92.52 \%$ of all upward moves, $90.86 \%$ of all lateral moves, and $90.12 \%$ of all downward moves (see Table 1).

The results of these multiepisode job shift models are reported in Table 2, where only estimates for the rates of upward and downward moves are displayed. The results of lateral moves are not presented because they "represent a mixture of gains and losses in job rewards other than those summarized by status" (Sorensen and Tuma, 1981:83).

The dependent variable is the number of months that a man spent in the labor market before he moved or before censoring occurred. This means that the clock that times career processes is the extent of an individual's general labor-force experience. Human capital economists often use this variable to measure general on-the-job training (Mincer, 1974). In the vacancy competition model, this variable is used as a measure of the gap between actual and expected rewards 


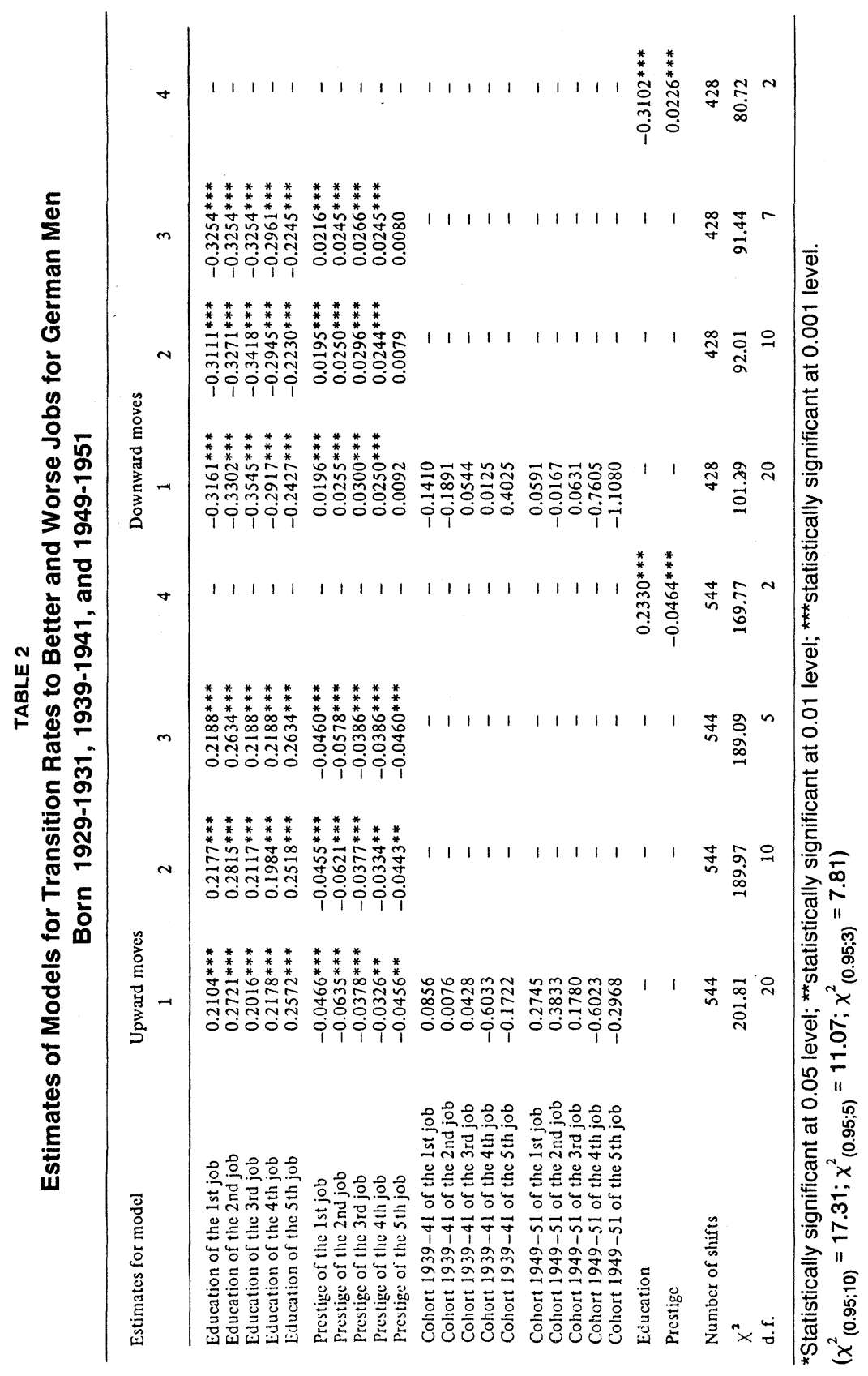


(Sorensen, 1984). With regard to the direction of job moves, Wegener's (1985) magnitude prestige scale defines prestige increases of $10 \%$ and greater as upward moves, prestige increases between 0 and $10 \%$ as lateral moves, and all prestige decreases as downward moves.

In Table 2, the relative magnitude of the effects of different variables (e.g., prestige or education) are not compared within models because they depend on the scale in which variables are measured. Instead, the significance level is used to indicate the statistical significance of the variables. In evaluating a model's performance, a likelihood ratio test also compares the model to a baseline (Blossfeld et al., 1988). The baseline for each directional move is the model without independent sariables. This test gives the chi-square values that are reported in Table 2.

On the basis of the human capital and vacancy competition theory, one can argue that the basic explanatory variables for rates of moving up or down are job rewards and personal resources (Tuma, 1985). Occupational prestige is used as a measure of job rewards, as measured here by Wegener's (1985) magnitude prestige scale (ranging from 22.0 to 186.6). Education is used as a measure of observed personal resources (Blossfeld, 1987a, 1987b). To control for cohort specific influences, dummy variables for the three birth cohorts also are included.

To estimate this multiepisode proportional hazard model, the program RATE (3.0) was used. Compared to BMDP, SAS, or GLIM, RATE allows one to specify both starting and ending times. Through this, one can include the specific location of any job spell within a person's career.

It will be useful to test step-by-step whether the variables have episode-changing influences on the job-shift rate. This procedure lets one begin with a complex model and then gradually introduce simplicity. By using RATE (3.0), this can be done using linear constraints.

Model 1 includes prestige, education, and birth cohort for each of the first five job episodes. For both upward and downward moves, this model performs significantly better than the baseline model. The positive episode-specific effects of education on upward moves are in accordance with a modified human capital theory (Tuma, 1985) and 
vacancy competition theory (Sorensen, 1981). The negative effects of prestige on upward moves can also be explained by the vacancy competition theory. According to Sorensen, opportunities for better jobs decline as the level of attainment already achieved increases.

The negative effects of education and the positive effects of prestige on downward moves are also in agreement with a modified form of the human capital theory (Tuma, 1985).

The effects of the birth cohort dummies are significant for neither upward nor downward moves (see Table 2). In Model 2 therefore, these variables are left out, and slightly smaller chi-square values for upward (189.97) and downward moves (92.01) are obtained while 10 degrees of freedom are saved in each model. The absolute magnitude of the episode-specific estimates of prestige and education also remain more or less unchanged.

According to both human capital theory and vacancy competition theory, one would expect constant effects of prestige and education across episodes. To test this hypothesis, the absolute magnitude of the episode-specific coefficients was compared in Model 2. For upward moves, the effects of education in the first $(\beta 1)$, third $(\beta 3)$, and fourth $(\beta 4)$ episodes are more or less the same, and the effects in the second $(\beta 2)$ and fifth $(\beta 5)$ episodes are also similar; for prestige, the effects in the first $(\beta 6)$ and fifth $(\beta 10)$ episode are comparable, as are the effects in the third $(\beta 8)$ and fourth $(\beta 9)$ episodes. For downward moves, the effects of education in the first $(\beta 1)$, second $(\beta 2)$, and third $(\beta 3)$ episodes are analogous; and for prestige, the effects in the second $(\beta 7)$ and fourth $(\beta 9)$ episodes are also similar. In Model 3, constraints for upward $(\beta 1=\beta 3=\beta 4 ; \beta 2=\beta 5)$ and downward moves $(\beta 1=\beta 2=$ $\beta 3 ; \beta 7=\beta 9$ ) were imposed to test the equality of these coefficients (Tuma, 1986). Table 2 shows that this model performs as well as Model $2(189.09 ; 91.44)$ for both upward and downward moves but saves 5 and 3 degrees of freedom, respectively. Hence, these constraints are justified.

Model 4 takes this a step further and estimates only episode-constant coefficients for education and prestige. Looking at the change in chi-square values, it can be seen that the difference is statistically significant at the 0.05 level for upward moves, but not for downward moves. This means that with respect to statistical criteria it is ap- 
propriate to use episode-constant coefficients only for downward moves, but not for upward moves. For upward moves, there is a statistically significant difference between the effects of education and prestige in different job episodes, a finding that should not be neglected.

\section{CONCLUSION}

In sociology, economics, or industrial engineering, the increasing availability of event history data permits the application of sophisticated statistical techniques to estimate economic and social processes. Frequently, this leads to general multistate-multiepisode models in which successive episodes represent durations in different states. This article showed how Cox models can be used to estimate these models. Using a large life history data set of German men born 1929-1931, 1939-1941, and 1949-1951, the estimation of a multiepisode proportional hazards model that controls for the time-dependent effect of labor-force participation was shown.

This analysis shows that for downward moves using an episodeconstant model it was justified, but for upward moves some statistically significant differences between the effects of education and prestige in different job episodes should not be neglected. Social scientists should, therefore, not only give more attention to the fact that social processes are usually multiepisode processes, but also should consider that the use of episode-constant coefficients in these models requires examining whether these coefficients are, in fact, statistically the same across episodes.

\section{REFERENCES}

BLOSSFELD, H.-P. (1987a) "Labor market entry and the sexual segregation of careers in the Federal Republic of Germany.” Amer. J. of Sociology 93: 89-118.

BLOSSFELD, H.-P. (1987b) "Entry into the labor market and occupational career in the Federal Republic of Germany. A comparison with American studies," pp. 86-115 in W. Teckenberg (ed.), Comparative Studies of Social Structure. New York: Armork. 
BLOSSFELD, H.-P. (1987c) "Zur repräsentativität der Sfb 3-lebensverlaufsstudie. Ein vergleich mit daten aus der amtlichen statistik." Allgemeines Statistisches Archiv 71: 126-144.

BLOSSFELD, H.-P., A. HAMERLE, and K. U. MAYER (1988) Event History Analysis. Hillsdale, NY: Lawrence Erlbaum.

BRÜCKNER, E, H.-P. KIRSCHNER, M. WEIDENBECK, and J. HOFFMEYER-ZLOTNIK (1984) Methodenbericht "Lebensverläufe." Mannheim: ZUMA.

CARROLL, G. R. and K. U. MAYER (1986) "Job-shift patterns in the Federal Republic of Germany: The effects of social class, industrial sector, and organizational size." Amer. Soc. Rev. 51: 323-341.

COX, D. R. (1972) "Regression models and life tables (with discussion)." J. of the Royal Stat. Society B 34: 187-220.

COX, D. R. (1975) “Partial likelihood." Biometrika 62: 269-275.

HAMERLE, A. (forthcoming) "Multiple-spell regression models for duration data." Applied Statistics 38.

HECKMAN, J. J. and B. SINGER (1984) “Econometric duration analysis." J. of Econometrics 24: 63-132.

KALBFLEISCH, J. D. and G. R. CARROLL (forthcoming) "Jobs and classes: structural constraints on career mobility." European Soc. Rev. Presented to the American Sociological Association, 26 August to 2 September 1986, New York City and Berlin.

KALBFLEISCH, J. D. and R. L. PRENTICE (1980) The Statistical Analysis of Failure Time Data. New York: Wiley.

MINCER, J. (1974) Schooling, Experience, and Earnings. New York: Columbia Univ. Press.

SORENSEN, A. B. (1983) "The structure of allocation to open and closed positions in social structure.” Zeitschrift für Soziologie 12: 203-224.

SORENSEN, A. B. (1984) "Interpreting time dependency in career processes," pp. 89-122 in A. Diekmann and P. Mitter (eds.), Stochastic Modelling of Social Processes. New York: Academic Press.

SORENSEN, A. B. and N. B. TUMA (1981) "Labor market structures and job mobility." Research in Social Stratification and Mobility 1: 67-94.

TUMA, N. B. (1985) "Effects of labor market structure on job-shift patterns," pp. 327-363 in J. J. Heckman and S. Singer (eds.), Longitudinal Analysis of Labor Market Data. Cambridge: Cambridge Univ. Press.

TUMA, N. B. (1986) "Invoking RATE. A draft of the documentation for RATE 3.0." (unpublished)

TUMA, N. B. and M. T. HANNAN (1984) Social Dynamics: Models and Methods. New York: Academic Press.

WEGENER, B. (1985) “Gibt es sozialprestige?” Zeitschrift für Soziologie 14: 209-235.

\footnotetext{
Hans-Peter Blossfeld is Senior Research Scientist at the Max Planck Institute for Human Development and Education in Berlin and faculty member (privatdozent) in sociology at the Free University, Berlin. His research interests include longitudinal studies and quantitative research methods, especially event history analysis, panel analysis, and life course analysis.
} 
Alfred Hamerle is Professor of Statistics, Faculty of Economics and Statistics, University of Konstanz, FRG. His research interests include developing dynamic statistical models for the analysis of event history and panel data. 